\title{
Economic Modelling of Chronic Kidney Disease: A Systematic Literature Review to Inform Conceptual Model Design
}

\author{
Daniel M. Sugrue ${ }^{1} \cdot$ Thomas Ward $^{1} \cdot$ Sukhvir Rai ${ }^{1} \cdot$ Phil McEwan $^{1} \cdot$ Heleen G. M. van Haalen ${ }^{2}$
}

Published online: 30 September 2019

(c) The Author(s) 2019

\begin{abstract}
Background Chronic kidney disease (CKD) is a progressive condition that leads to irreversible damage to the kidneys and is associated with an increased incidence of cardiovascular events and mortality. As novel interventions become available, estimates of economic and clinical outcomes are needed to guide payer reimbursement decisions.

Objective The aim of the present study was to systematically review published economic models that simulated long-term outcomes of kidney disease to inform cost-effectiveness evaluations of CKD treatments.

Methods The review was conducted across four databases (MEDLINE, Embase, the Cochrane library and EconLit) and health technology assessment agency websites. Relevant information on each model was extracted. Transition and mortality rates were also extracted to assess the choice of model parameterisation on disease progression by simulating patient's time with end-stage renal disease (ESRD) and time to ESRD/death. The incorporation of cardiovascular disease in a population with CKD was qualitatively assessed across identified models.

Results The search identified 101 models that met the criteria for inclusion. Models were classified into CKD models $(n=13)$, diabetes models with nephropathy $(n=48)$, ESRD-only models $(n=33)$ and cardiovascular models with CKD components $(n=7)$. Typically, published models utilised frameworks based on either (estimated or measured) glomerular filtration rate (GFR) or albuminuria, in line with clinical guideline recommendations for the diagnosis and monitoring of CKD. Generally, two core structures were identified, either a microsimulation model involving albuminuria or a Markov model utilising CKD stages and a linear GFR decline (although further variations on these model structures were also identified). Analysis of parameter variability in CKD disease progression suggested that mean time to ESRD/death was relatively consistent across model types (CKD models 28.2 years; diabetes models with nephropathy 24.6 years). When evaluating time with ESRD, CKD models predicted extended ESRD survival over diabetes models with nephropathy (mean time with ESRD 8.0 vs. 3.8 years). Discussion This review provides an overview of how CKD is typically modelled. While common frameworks were identified, model structure varied, and no single model type was used for the modelling of patients with CKD. In addition, many of the current methods did not explicitly consider patient heterogeneity or underlying disease aetiology, except for diabetes. However, the variability of individual patients' GFR and albuminuria trajectories perhaps provides rationale for a model structure designed around the prediction of individual patients' GFR trajectories. Frameworks of future CKD models should be informed and justified based on clinical rationale and availability of data to ensure validity of model results. In addition, further clinical and observational research is warranted to provide a better understanding of prognostic factors and data sources to improve economic modelling accuracy in CKD.
\end{abstract}

Electronic supplementary material The online version of this article (https://doi.org/10.1007/s40273-019-00835-z) contains supplementary material, which is available to authorized users.

Daniel M. Sugrue

daniel.sugrue@heor.co.uk

1 Health Economics and Outcomes Research Limited, Rhymney House, Unit A Copse Walk, Cardiff Gate Business Park, Cardiff CF23 8RB, UK

2 AstraZeneca, Gothenburg, Sweden

\section{Introduction}

Chronic kidney disease (CKD) is a progressive condition with heterogenous aetiology that leads to irreversible damage to the kidneys and is associated with an increased incidence of cardiovascular events and mortality [1-6]. The age-standardised global prevalence of CKD stages 1-5 among adults aged $\geq 20$ years in 2010 was approximately $10.4 \%$ in men and $11.8 \%$ in women and continues to rise, fuelled by ageing populations and the increasing incidence 


\section{Key Points for Decision Makers}

This review provides an overview of how chronic kidney disease (CKD) is typically modelled, with glomerular filtration rate (GFR) and albuminuria, respectively, typically utilised as the key prognostic factor within CKD and diabetes model frameworks.

Most of the models identified were Markov models and/ or utilised input data at cohort mean levels, and many of the current methods did not explicitly consider patient heterogeneity or underlying disease aetiology, except for diabetes, providing limited clinical rationale for the choice of model design.

Given the heterogenous nature of individual CKD patients' characteristics and clinical prognoses, a model structure designed around the prediction of individual patients' GFR trajectories may be preferred over cohortbased modelling frameworks when simulating patients with CKD. However, model choice should be informed and justified based on clinical rationale and availability of data to ensure validity of model results.

of diabetes and hypertension [7, 8]. Currently, more than two million patients with CKD worldwide have been estimated to require renal replacement therapy (RRT) through dialysis or kidney transplantation [9]. In addition, the humanistic and economic burdens of CKD increase significantly as renal function declines, and patients at CKD stage 3 or above are at 1-14 times higher risk for cardiovascular and overall mortality compared with the general population [4]. Moreover, patients with CKD often experience healthrelated quality of life (HRQoL) impairments and increased healthcare costs $[4,10]$. Because of the need for intensive treatment, the largest costs and impacts on HRQoL are incurred when patients reach CKD stage 5 (glomerular filtration rate $[\mathrm{GFR}]<15 \mathrm{~mL} / \mathrm{min} / 1.73 \mathrm{~m}^{2}$ ) or end-stage renal disease (ESRD), which is irreversible and where patients often require RRT [11, 12].

Cardiovascular disease (CVD) and CKD are closely interrelated, and the reduction of systolic blood pressure, blood glucose and other cardiovascular risk factors via drug treatment and/or lifestyle modifications are key components of CKD management. Renin-angiotensin-aldosterone system inhibitors, which confer both cardio- and reno-protective benefits through their anti-hypertensive effect, have been the cornerstone of CKD treatment for many years [13, 14] and are often prescribed in addition to statins and anti-platelet therapy [15-19]. While the risk of developing CKD of certain aetiologies can be reduced through medical and/or lifestyle interventions, others cannot, and the main goals of CKD management are to delay the rate of progression to ESRD to limit cardiovascular risk, preserve HRQoL and restrain healthcare costs. However, the underlying aetiology is often predictive of both the likely treatment strategy and the clinical prognosis.

As novel treatments for CKD become available and require evaluation for inclusion into drug formularies, estimates of economic (optimisation of budget usage) and clinical (maximisation of population benefits) outcomes are needed to guide payer reimbursement decisions. Given the significant costs associated with ESRD management, the rate of progression to ESRD is a significant determinant of cost effectiveness. However, the long-term outcomes of CKD, such as ESRD incidence, are not always sufficiently captured over the duration of clinical studies, and time to ESRD often varies widely between patients [20], making extrapolation necessary yet challenging. The choice of model structure may also play a key role in the validity of these extrapolations, underlining the importance of understanding the implications of selecting a particular conceptual modelling approach. With these challenges in mind, the aim of the present review was to systematically review published economic models that simulated long-term outcomes of kidney disease to qualitatively describe the chosen model designs, rates of CKD progression and the incorporation of CVD in a population with CKD.

\section{Methods}

\subsection{Literature Search and Data Extraction}

This review was conducted according to the PRISMAP (Preferred Reporting Items for Systematic reviews and Meta-Analyses Protocols) guidelines [21]. A search strategy was devised using a specified set of search terms for each database (Tables 1-6 in Resource 1 of the Electronic Supplementary Material [ESM]). In brief, the search was designed to identify economic modelling publications that included adult patients with/without CKD in whom CKD progression was evaluated.

Searches were conducted in MEDLINE, Embase, the Cochrane Library and EconLit on 10 November 2017. To identify additional publications that may have modelled progression of kidney disease as part of another disease model, we conducted a supplementary search in MEDLINE to identify systematic literature reviews detailing economic models for diabetes or hypertension. We also searched the websites of the following health technology assessment agencies: UK National Institute for Health and Care Excellence, Canadian Agency for Drugs and Technologies in Health and the Australian Pharmaceutical Benefits Advisory Committee. 
Bibliographies of eligible articles were also searched for potential publications of interest to the review.

Titles and abstracts of the identified citations were screened by a single reviewer following specific PICOS (population, interventions, comparators, outcomes and study design) eligibility criteria (Table 7 in Resource 1 of the ESM). In brief, studies were included if they described an economic model of adult patients with/without CKD in whom CKD progression was evaluated. The model structure must have included the progression of kidney disease and any health economic outcome, including (but not restricted to) quality-adjusted life-years, incremental cost-effectiveness ratios, life-years gained or costs. Studies were limited to English language only. Abstracts/titles that did not meet the eligibility criteria were excluded; full-text publications were obtained for the remaining citations. The screening process was repeated using the PICOS criteria for full-text articles to obtain a final set of included publications that described economic models characterising CKD progression, regardless of the intervention or comparator being assessed.

Information on model characteristics (including structure, perspective, health states and disease setting), disease progression, event utility, costs, sensitivity analyses, drivers of cost effectiveness, validation procedures and model limitations were extracted from all included publications.

\subsection{Analysis of Time to End-Stage Renal Disease (ESRD) or Death}

Where reported, transition and mortality rates were extracted from publications to qualitatively describe the choice of model parameterisation on disease progression, such as time to and time with ESRD. To facilitate comparison of rates of CKD disease progression, transition rates from each eligible published model were used to estimate time to ESRD or death (ESRD/death) and time with ESRD within a simple Markov model framework. Such analyses were undertaken individually for each model and limited to decision trees and Markov models that reported sufficient data to allow such analyses. Published models were excluded from these analyses if transition rate data were not reported in sufficient detail or if regression-based simulation approaches were modelled because of their complexity. Models were also excluded if they were designed to model a horizon of $<10$ years.

Health states were defined based on those reported in each individual model publication (Fig. 1 in Resource 2 of the ESM). Where possible, analyses were initiated with a standard patient profile, defined as patients aged 50 and from CKD stage 1, normo-albuminuria or earliest disease stage modelled, to provide a consistent reference point. The cycle length reported in each publication was utilised and the time to ESRD/death and time with ESRD subsequently calculated by estimating the time spent in each of the modelled health states. A maximum horizon of 50 years (up to the age of 100) was modelled across all analyses to limit the uncertainty between modelled outcomes.

\section{Results}

\subsection{Summary of Included Models}

The search identified 2382 citations, excluding any duplicate publications (Fig. 1). During the screening of titles and abstracts, a further 1880 citations were excluded. Upon fulltext assessment of the remaining 502 articles, 101 models met criteria for inclusion, which were classified into CKD models $(n=13)$, diabetes models with nephropathy $(n=48)$, ESRD-only models $(n=33)$ and cardiovascular disease (CVD) models with CKD components $(n=7)$.

Models were assessed using the Consolidated Health Economic Evaluation Reporting Standards (CHEERS) checklist for economic evaluation of health interventions, which assesses whether all key elements of model structure, parameterisation and approach have been clearly reported [22]. In 15 (15\%) models, all elements of the CHEERS checklist were included, with 79 (78\%) models including $\geq 80 \%$ of applicable elements (Resource 3 of the ESM). Often there was no single table of modelling assumptions; instead, structural or other assumptions underpinning the models were reported in relevant sections of the publication. The most infrequently reported elements were conflicts of interest (47\%), measurement and valuation of preferencebased outcomes (34\%) and sources of funding (30\%).

\subsection{Chronic Kidney Disease (CKD) Models}

\subsubsection{Framework and Disease Pathway}

In 13 publications that described unique CKD models, a Markov model was most commonly used (ten publications [77\%]) [23-32], including two models that were developed to inform clinical and policy decisions in improving CKD care: the Chronic Kidney Disease Model [31] and the SHARP CKD-CVD model [32] (Table 1). A further model, the CKD Health Policy Model, was also developed to inform policy-making decisions and used a microsimulation approach [33]. Across models, CKD was generally described and modelled as a single disease. Other aetiologies included CKD and hypertension [26] and CKD and hepatitis $\mathrm{C}$ virus genotype [25], but a general population of individuals was more common [23, 24, 27, 33]. Where reported, the underlying disease aetiologies are described in Supplementary Table 8 (Resource 4 of the ESM). Also, an overview of the model settings, health states related to kidney disease, approach to model CKD progression, approach 
Fig. 1 PRISMA (Preferred Reporting Items for Systematic Reviews and Meta-Analyses) diagram showing the modelselection process. $C K D$ chronic kidney disease, $C V D$ cardiovascular disease, ESRD end-stage renal disease, HTA health technology appraisal
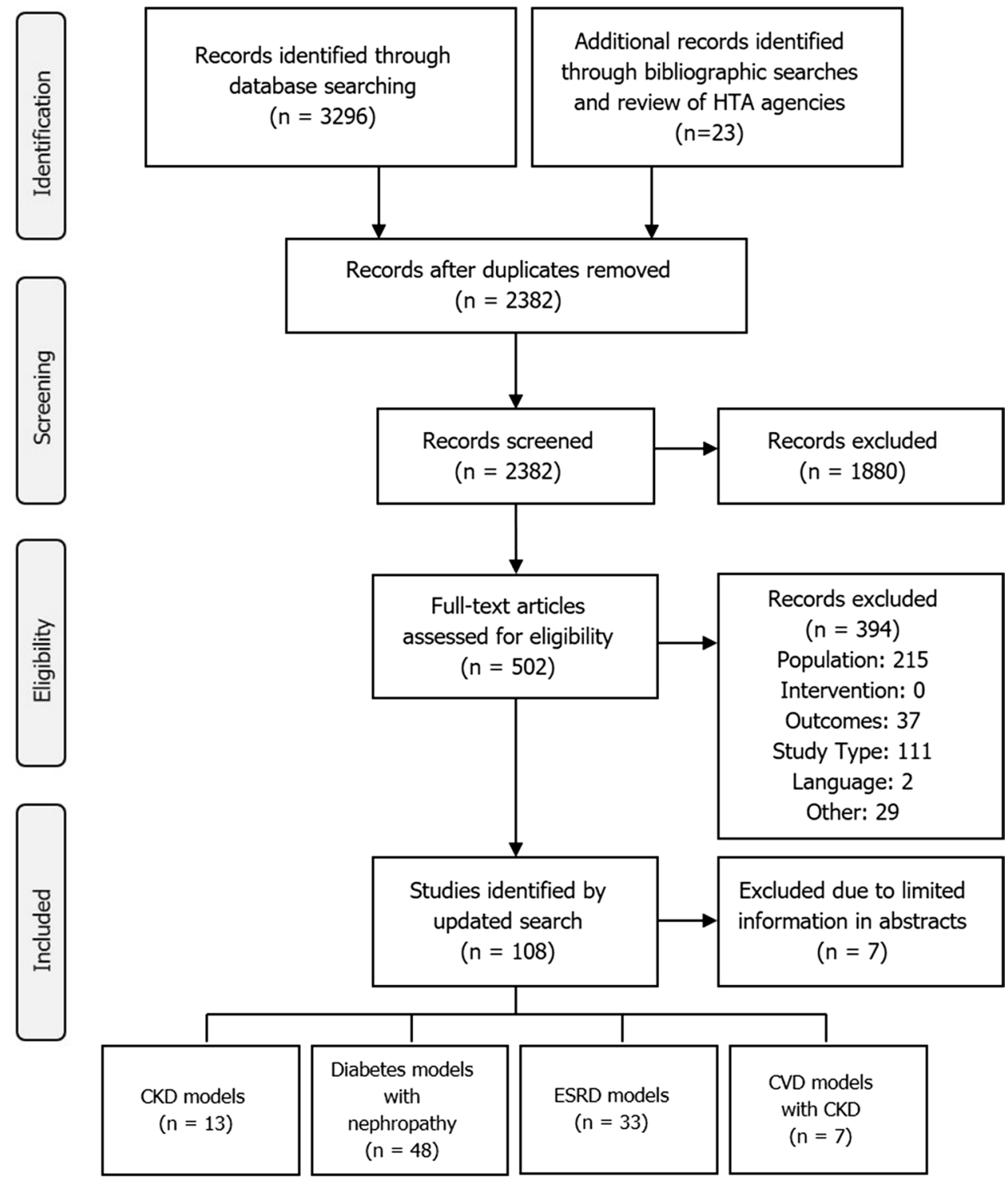

to modelling cardiovascular events, discounting, sensitivity analyses, drivers of cost effectiveness and approach to model validation is available in Supplementary Tables 8-10 (Resource 4 of the ESM).

In general, models were based on a core structure defined by health states for CKD according to (estimated or measured) GFR, in line with stratifications in the National Kidney Foundation Kidney Disease Outcomes Quality Initiative (NKF KDOQI) guidelines [34] (Fig. 2). However, four models did not apply this structure [23, 27, 35, 36]. Boersma et al. [23] modelled CKD according to the presence of albuminuria (normoalbuminuria, microalbuminuria and macroalbuminuria). Ferguson et al. [27] divided model states into normal kidney function; low, intermediate and high risk of progression to kidney failure; being on dialysis; and surviving with a kidney transplant. Howard et al. [36] simulated the annual progression of patients from the development of risk factors (specifically hypertension, proteinuria and diabetes [with no albuminuria, microalbuminuria or macroalbuminuria]) through CKD stage 1-4 to ESRD and RRT. In most studies, patients initiated RRT with dialysis and had the option to receive a transplant. However, a number of studies did not differentiate between type of RRT, instead defining RRT as dialysis or transplant. For hypertension and proteinuria states, patients in this modelling study had an annual probability of progressing to ESRD requiring RRT, where CKD progression was not explicitly modelled. Finally, Okubo et al. [35] formulated a Markov model using separate sub-models for patients with CKD with and without diabetes. In both, kidney disease was modelled as the probability of ESRD. All models included transition to death. 
Table 1 Summary of model structural framework and the method of disease progression across identified chronic kidney disease and diabetes models

\begin{tabular}{|c|c|c|c|c|}
\hline$N$ & Model structure & Model structure sub-category & CKD progression (as described by model publication) & References \\
\hline \multicolumn{5}{|c|}{ CKD models $(N=13)$} \\
\hline \multirow[t]{9}{*}{9} & \multirow[t]{9}{*}{ Markov model } & Multistate Markov model & Transition probabilities & {$[23]$} \\
\hline & & Markov model & GFR decline & {$[24]$} \\
\hline & & Semi-Markov model & Transition probabilities & {$[25]$} \\
\hline & & Markov model & Estimated GFR decline, transition probabilities & {$[26]$} \\
\hline & & Markov model & Transition probabilities & {$[27]$} \\
\hline & & Markov model & Estimated GFR decline, transition probabilities & {$[28]$} \\
\hline & & Markov model & Transition probabilities & {$[30]$} \\
\hline & & Markov model & Transition probabilities & [29] \\
\hline & & Markov model & Risk equations & {$[32]$} \\
\hline 1 & Simulation model & Microsimulation model & Estimated GFR decline & [163] \\
\hline \multirow[t]{3}{*}{3} & \multirow[t]{3}{*}{ Combination } & Markov model and Monte Carlo simulation & Relative risk of progression & [36] \\
\hline & & Decision tree and Markov model & Transition probabilities & {$[35]$} \\
\hline & & Markov model and Monte Carlo simulation & GFR decline & {$[31]$} \\
\hline \multicolumn{5}{|c|}{ Diabetes models $(N=48)$} \\
\hline \multirow[t]{25}{*}{25} & \multirow[t]{25}{*}{ Markov model } & Markov model & Transition probabilities & [115] \\
\hline & & Markov model & Transition probabilities & [118] \\
\hline & & Markov model & Transition probabilities & {$[90]$} \\
\hline & & Markov model & Transition probabilities & [79] \\
\hline & & Markov model & Transition probabilities & [98] \\
\hline & & Markov model & Transition probabilities & [116] \\
\hline & & Markov model & Transition probabilities & {$[53]$} \\
\hline & & Markov model & Creatinine clearance decline & {$[124]$} \\
\hline & & Markov model & Transition probabilities & [92] \\
\hline & & Markov model & Transition probabilities & {$[100]$} \\
\hline & & Markov model & Transition probabilities & [89] \\
\hline & & Markov model & Transition probabilities & [164] \\
\hline & & Markov model & Transition probabilities & {$[85]$} \\
\hline & & Markov model & Transition probabilities & {$[120]$} \\
\hline & & Markov model & Transition probabilities & 105 \\
\hline & & Markov model & Transition probabilities & [88] \\
\hline & & Markov model & Transition probabilities & [110] \\
\hline & & Markov model & $\mathrm{HbA}_{1 \mathrm{c}}$ levels & {$[86]$} \\
\hline & & Markov model & Transition probabilities & {$[102]$} \\
\hline & & Markov model & Transition probabilities & {$[103]$} \\
\hline & & Markov model & Risk equations & [104] \\
\hline & & Markov model & Transition probabilities & [113] \\
\hline & & Markov model & Transition probabilities & [74] \\
\hline & & Semi-Markov model & Transition probabilities & [117] \\
\hline & & Semi-Markov model & Transition probabilities & {$[107]$} \\
\hline \multirow[t]{9}{*}{13} & \multirow[t]{9}{*}{ Simulation model } & Discrete-event simulation model & Transition probabilities & {$[121]$} \\
\hline & & Microsimulation model & Risk equations & [109] \\
\hline & & Microsimulation model & Incidence rates & [91] \\
\hline & & Discrete-event simulation model & Risk equations & {$[95]$} \\
\hline & & Microsimulation model & Risk equations & {$[93]$} \\
\hline & & Microsimulation model & Transition probabilities & {$[83]$} \\
\hline & & Object-oriented simulation model & Risk equations & [112] \\
\hline & & Microsimulation model & Risk equations & [99] \\
\hline & & Discrete-event simulation model & Transition probabilities & {$[101]$} \\
\hline
\end{tabular}


Table 1 (continued)

\begin{tabular}{|c|c|c|c|c|}
\hline$N$ & Model structure & Model structure sub-category & CKD progression (as described by model publication) & References \\
\hline \multirow{6}{*}{2} & \multirow{6}{*}{ Decision tree } & Microsimulation model & Transition probabilities & [96] \\
\hline & & Discrete-event simulation model & Transition probabilities & {$[84]$} \\
\hline & & Monte Carlo simulation model & Transition probabilities & {$[87]$} \\
\hline & & Microsimulation model & Not reported & {$[106]$} \\
\hline & & Decision tree & Creatinine clearance decline & [96] \\
\hline & & Decision tree & Probabilities & {$[82]$} \\
\hline \multirow[t]{8}{*}{8} & \multirow[t]{8}{*}{ Combination } & Markov model and Monte Carlo simulation & Transition probabilities & [94] \\
\hline & & Markov model and Monte Carlo simulation & Transition probabilities & [119] \\
\hline & & Markov model and microsimulation & Transition probabilities & {$[122]$} \\
\hline & & Semi-Markov model and Monte Carlo simulation & Transition probabilities & {$[81]$} \\
\hline & & Markov model and Monte Carlo simulation & Hazard rate (per year) & [97] \\
\hline & & Markov model and Monte Carlo simulation & Risk equations & [114] \\
\hline & & Markov model and Monte Carlo simulation & Transition probabilities & {$[80]$} \\
\hline & & Decision tree and Markov model & Transition probabilities & [111] \\
\hline
\end{tabular}

Decision tree: Defined as a cohort-level model that uses a tree-like model of decisions and their possible consequences, where transitions are limited to those specified by the particular nodes included in the decision tree. Markov model: A more fluid extension of the decision tree principle, defined as a type of cohort-based mathematical model containing a finite number of mutually exclusive health states, with time periods of uniform length, in which the probability of movement from one state to another depends on the current state. Semi-Markov model: As a Markov model but incorporating a time-dependency factor whereby transition rates are dependent on the time spent in a health state and are, thus, not constant. Multi-state Markov model: As a Markov model but has explicitly described the calculation of transition rates as accounting for dependencies between events. Simulation model: Defined as a patient-level model in which patient disease progression is simulated individually and where health states are not modelled as mutually exclusive. Microsimulation model: As a simulation model but providing more granularity/detail (e.g. where a simulation model may model ESRD as a single state, a microsimulation model may model health states within the ESRD state such as dialysis or transplant). Object-oriented simulation model: Defined as a person-by-person, object-by-object simulation, spanning from biological details to the care processes, logistics, resources and costs of healthcare systems. Monte-Carlo simulation model: Defined as a form of modelling where model inputs are drawn from distributions and are not treated as fixed values, with the model run multiple times to provide a probabilistic distribution of results. Discrete-event simulation: Discrete-event simulation is a computer-modelling technique used in economic evaluation of health interventions in which individual patient experience is simulated over time, and events occurring to the patient and the consequences of such events are tracked and summarised. Unlike other models, in discrete-event simulation, movements between patients' health states are usually driven by events that may occur at varying times (rather than during cycles of fixed length), and time-to-event distributions are required for each event. Event likelihoods are driven by individual patient characteristics, which are recorded at baseline and may be updated as the patient experience (events, new health states) accumulates

$C K D$ chronic kidney disease, ESRD end-stage renal disease, $G F R$ glomerular filtration rate, $H b A_{1 c}$ glycated haemoglobin

There were four common variations to the core structure of reported models (Fig. 2): (1) modelling CKD stage 3 as a single health state or stratified into CKD stage $3 \mathrm{a}$ and $3 \mathrm{~b}$; (2) the initial CKD stage (i.e. whether patients began in early or advanced CKD stages); (3) presenting CKD stage 5 as a single state (ESRD) or stratified into non-dialysis ESRD, dialysis or kidney transplant; and (4) using separate sub-models for diabetes and CKD that informed the overall model. Where reported, ESRD models defined ESRD as (measured or estimated) GFR $<15 \mathrm{~mL} / \mathrm{min} / \mathrm{m}^{2}$; the initiation rules for RRT were less consistent. Four models assumed initiation of RRT (dialysis or transplant) immediately after the incidence of ESRD [29, 30, 32, 36], whereas four models assumed a delay in the initiation of RRT; one model assumed RRT was initiated 1 year after ESRD incidence [33], two models initiated RRT at a GFR $<10 \mathrm{~mL} / \mathrm{min} / \mathrm{m}^{2}[28,31]$ and one assumed a static transition probability to RRT [25].

\subsubsection{Characterisation of CKD Progression}

In summary, five models (38\%) predicted CKD outcomes based on rates of annual GFR decline, four models (31\%) used GFR transition probabilities and two models (15\%) used albuminuria transition probabilities.

Models based on GFR health states predicted CKD outcomes based on rates of GFR decline or transition probabilities. Erickson et al. [26] used published observations from large observational cohorts of comparable patient populations to estimate the mean annual rate of estimated GFR decline. This annual rate was applied to all CKD stages (progression from stage 3 a to stage $5 \mathrm{CKD}$ ). Boulware et al. [24] and the CKD Health Policy Model [33] modelled progression through CKD stages based on GFR decline and albuminuria status. In the CKD Health Policy Model, a person would be assigned an initial estimated GFR and 


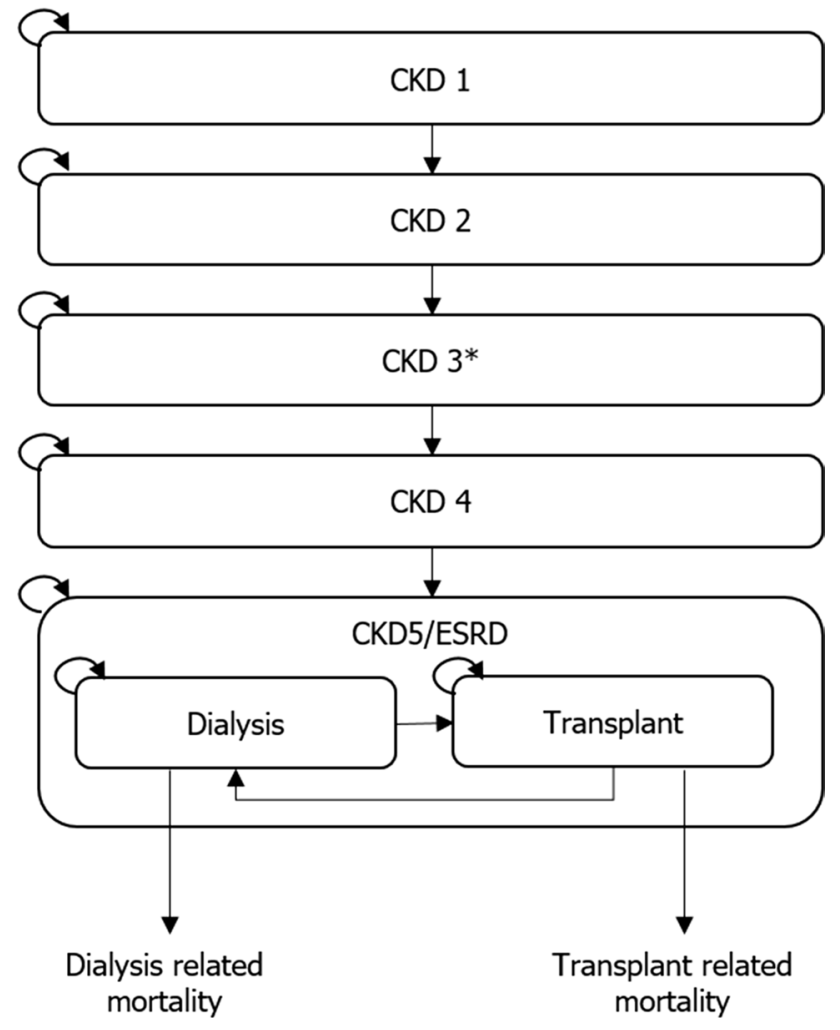

Fig. 2 Example of the core model structure of chronic kidney disease models. $C K D$ chronic kidney disease, ESRD end-stage renal disease. ${ }^{*}$ CKD stage 3 may be further stratified into CKD stage $3 a$ and CKD stage $3 \mathrm{~b}$. Note: Patients could progress to death from any health state

subsequently experience annual decreases in estimated GFR based on rates derived from a clinical trial by Boulware et al. [24] that varied according to CKD stage, presence of albuminuria and diabetes, and hypertension status. The model by Levy et al. [28] modelled the functioning kidney as a single health state; estimated GFR was assumed to decline linearly from estimated GFR 3a until ESRD. The Chronic Kidney Disease model [31] utilised GFR changes based upon a distribution derived from an analysis of a primary dataset of patients with CKD followed for 5 years [37] that was adjusted for age, race, current CKD stage, hypertension, diabetes, proteinuria, and CVD.

Four models $[25,27,29,30]$ utilised transition probabilities between CKD stages to model (estimated or measured) GFR decline. Elbasha et al. [25] derived probabilities from a targeted review of the published literature, Ferguson et al. [27] calculated probabilities based on estimated GFR values and adapted longitudinal data on CKD progression and regression rates from the indigenous cohort within the Alberta Kidney Disease Network [38], and Nuijten et al. [29, 30] used a published study by Keith et al. [39] to derive transition probabilities.
In the two models $[23,36]$ that used albuminuria states to model CKD progression, annual transition probability and mortality rates differed by proteinuria stage and were based on data from the PREVEND (Prevention of Renal and Vascular End Stage Disease) study [40].

Within the SHARP CKD-CVD policy model, the CKD sub-model utilised participant data from the SHARP (Study of Heart and Renal Protection) trial-a randomised trial assessing the effects of lowering low-density lipoprotein cholesterol in patients with CKD-to inform disease progression [32, 41]. For each year of follow-up, data collected during that year were used to categorise participants into respective health states (one of five CKD states, on maintenance dialysis, or having undergone a kidney transplant). Participants not receiving RRT could progress to (or remain in) any CKD category, with transition probabilities estimated using multivariate multinomial logistic regression.

Two models predicted improvement in health over time (e.g. CKD stage 2 to stage 1): Boersma et al. [23] and Ferguson et al. [27] allowed patients to transition to improved states of health during the model horizon, the former through transitions between macro-, micro- and normo-albuminuria, and the latter between CKD stages 1-4.

Four models [25-28] adapted mortality rates from baseline populations using age- and sex-specific life tables from the country of study and modified them using relative risks or hazard ratios to derive CKD stage-specific all-cause mortality. Another approach was to derive mortality rates from large cohort studies [23, 29,30]. Where modelled, mortality rates for those surviving with a transplant or those on dialysis, as well as the chance of graft failure, were taken from national registries.

\subsection{ESRD-Only Models}

Rather than modelling disease progression through its full spectrum (CKD stage 1-5), 33 models described an ESRD population (e.g. CKD stage 5 or a dialysis population). Across ESRD-only models, the costs and benefits of different dialysis modalities was the primary outcome assessed [42-51]. In addition, the cost effectiveness of treating hyperphosphatemia or anaemia, secondary conditions associated with CKD progression, were commonly modelled (e.g. use of sevelamer with calcium carbonate for patients receiving dialysis with hyperphosphatemia [52]).

Of these models, 19 (58\%) modelled patients from the explicit state of dialysis or transplant, and the remaining $14(42 \%)$ initiated patients from a pre-dialysis health state with or without ESRD, including health states describing pre-dialysis CKD stage 5 and other broader CKD health state definitions. Most models focusing on ESRD only used Markov modelling techniques (28 publications [42-45, 47, $48,50-71]$ ); among the remaining models, one [49] used a 
decision tree and another a microsimulation approach [46], and two further models used a Markov model with a decision tree component $[58,72]$. An overview of the models is provided in Supplementary Tables 11-13 (Resource 5 of the ESM).

In general, models employed a core structure based on four health states: pre-dialysis, dialysis, transplantation and death. The majority of models assumed the initiation of dialysis or transplantation at model initiation, with only two notable exceptions, the models by Shechter et al. [73] and Lee et al. [46], where dialysis was not initiated until GFR thresholds of $<10$ and $<9 \mathrm{~mL} / \mathrm{min} / \mathrm{m}^{2}$, respectively. Within the dialysis state, patients could switch between haemodialysis and peritoneal dialysis modalities. Patients could progress from the pre-dialysis state to dialysis or directly to kidney transplant; they could also reach the transplant state through progression from the dialysis state. Upon transplant, patients could remain in continued graft function or experience graft failure, whereby they returned to the dialysis state.

There were two common variations to this structure: first, models differed in the approach to modelling dialysis and, second, they differed on how kidney function was modelled post-transplant. Dialysis was modelled as a single health state $[44,51,52,55,66,68,71,74]$, as two separate health states for haemodialysis and peritoneal dialysis [45, 48-50, $56,58,62,63,67]$, as three separate health states [47, 65], or as four separate health states $[43,70]$ that included home haemodialysis, satellite haemodialysis, hospital haemodialysis and continuous ambulatory peritoneal dialysis [43]. Two models reported the use of five different dialysis modalities $[42,57]$. Regarding graft function post-transplant, the majority of models included a rate of graft failure, upon which patients would return to the dialysis health state. Four models included a post-transplant health state [44, 46, 60, 65].

In general, ESRD-only models used various forms of transition rates to model the proportion of pre-dialysis patients progressing to dialysis or transplant, as described briefly in Supplementary Tables 10-19 (Resources 5-7 of the ESM) [42-45, 47-52, 54, 56, 57, 60, 62-67, 70, 75]. These were usually derived using data obtained from national renal registries relevant to the country perspective of the model, such as the Norwegian Renal Registry [76], the UK Renal Registry [75] or the Dutch Renal Replacement Registry [77].

\subsection{Diabetes Models with Nephropathy}

\subsubsection{Framework and Disease Pathway}

Diabetic nephropathy is a microvascular complication of diabetes and a leading cause of ESRD [78]. In total, 48 unique diabetes models were identified that modelled nephropathy using two different approaches. The first approach modelled diabetes through estimated glycated haemoglobin $\left(\mathrm{HbA}_{1 \mathrm{c}}\right)$ change with time. The models included a sub-model of nephropathy as a long-term complication of diabetes; other complications included retinopathy and neuropathy. The second approach exclusively modelled the progression of nephropathy in diabetic patients through albuminuria health states, without modelling diabetes progression or $\mathrm{HbA}_{1 \mathrm{c}}$. Twelve (25\%) were models of type 1 diabetes mellitus (T1DM) [74, 79-89], 22 (46\%) were models of type 2 diabetes mellitus (T2DM) [90-111] and seven (16\%) were models of both T1DM and T2DM [112-117]. The remaining models evaluated either diabetes with hypertension [118-120], prediabetes [121, 122], or diabetes with more advanced kidney disease $[123,124]$. The majority of models were Markov $(n=25[52 \%])$ or microsimulation $(n=7[15 \%])$ models (Table 1). An overview of the models is provided in Supplementary Tables 14-16 (Resource 6 of the ESM).

Irrespective of the type of diabetes, most models applied a core sub-model structure of diabetic nephropathy based on a disease pathway of five health states: no nephropathy (or normoalbuminuria), microalbuminuria, macroalbuminuria (or gross proteinuria), ESRD (comprising dialysis or transplant as absorbing states) and death (Fig. 3). Some models also included a health state of macroalbuminuria with impaired (estimated or measured) GFR.

Generally, in models focusing on diabetic nephropathy, patients could progress from no nephropathy to microalbuminuria, from which they could either return to no nephropathy or progress to macroalbuminuria with or without impaired GFR. Once in a state of macroalbuminuria, with or without impaired GFR, patients could progress to ESRD. Across all diabetic nephropathy models, RRT (dialysis or transplant) was not explicitly defined in 15 models and not modelled entirely in nine models. When RRT was included in a model, it was typically initiated upon the incidence of ESRD (22 models) or after the first year of ESRD (one model). Patients with ESRD could usually progress from dialysis to transplant and, following graft failure, from transplant to dialysis; both the dialysis and the transplant health states were associated with an increased risk of mortality.

\subsubsection{Characterisation of CKD Progression}

Generally, in T1DM models, transition rates or transition probabilities informed nephropathy progression, with the source of disease progression commonly derived from the DCCT (Diabetes Control and Complications Trial), a major clinical trial conducted from 1983 to 1993 [125] or the randomised clinical trial of captopril for the inhibition of diabetic nephropathy [126]. Similarly, T2DM models tended to use transition rates or transition probabilities to model the progression of diabetic nephropathy. Two studies, the USRDS (United States Renal Data System) national database and the UKPDS (UK Prospective Diabetes Study), were 
used to inform transition rates or probabilities across many of the models. The JADE model [95], UKPDS OM1 model [96], UKPDS OM2 model [99] and the IHE cohort model [104] developed risk equations using data mainly derived from the UKPDS, among other sources.

Models including both T1DM and T2DM disease states also applied transition rates or transition probabilities to predict diabetic nephropathy progression. Information was generally sourced from different studies to inform separate rates/probabilities for T1DM and T2DM. The EAGLE [114] and CORE [115] diabetes models with CKD components used separate sources for ESRD only (T1DM [127], T2DM [128]), with the latter model employing three additional studies $[126,129,130]$ to inform T2DM transition probabilities. Furthermore, in the CORE diabetes model, patients were screened for the presence of micro- or macroalbuminuria; once either was detected, they could be treated with angiotensin-converting enzyme inhibitors. Patients in receipt of angiotensin-converting enzyme inhibitors progressed at a different rate from those not receiving these medications.

In all models, patients in the ESRD health state received dialysis or renal transplant. The decision to transition to dialysis was typically based on a weighted average of dialysis modalities (haemodialysis and peritoneal dialysis) that either reflected country-specific treatment patterns or were derived from contemporary studies. The rate at which patients progressed from dialysis to transplant or from transplant to

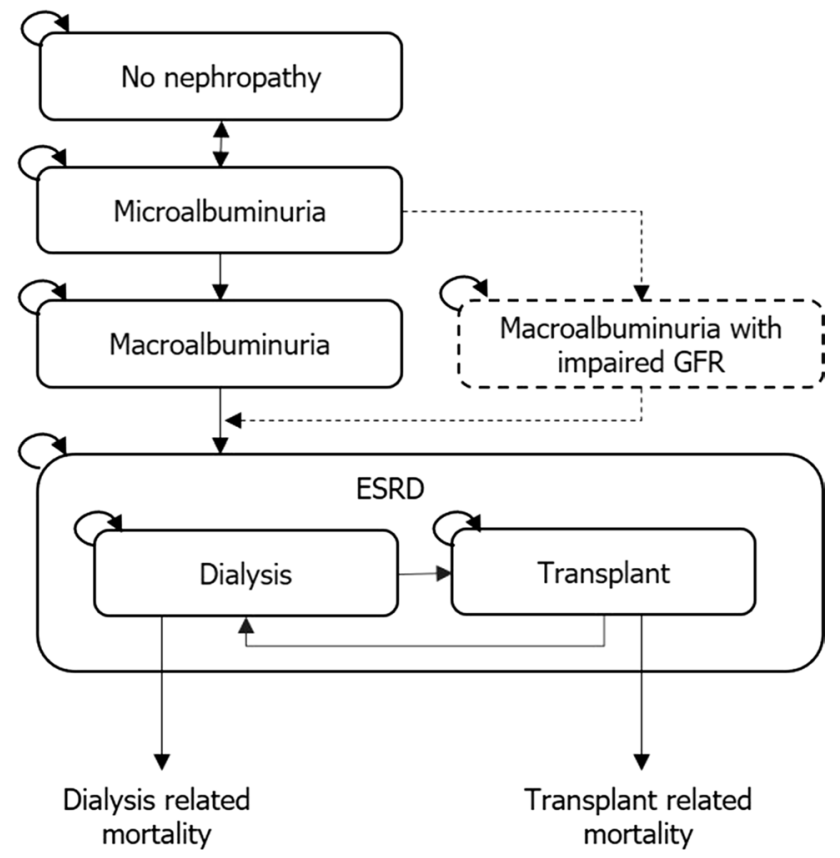

Fig. 3 Example of the core model structure of the nephropathy component of diabetes models with nephropathy. $C K D$ chronic kidney disease, ESRD end-stage renal disease, GFR glomerular filtration rate. Note: Patients could progress to death from any health state dialysis, and the mortality risk multiplier attributable ESRD, were typically informed by country-specific registry data.

\subsection{Cardiovascular Morbidity and Mortality}

Given the interrelationship between CKD and CVD, the incorporation of cardiovascular morbidity and mortality was assessed across identified models. In CKD models, the most commonly reported cardiovascular events were myocardial infarction (five models [23, 25, 26, 31, 33]) and stroke (four models [25, 26, 31, 33]). In two models, the Framingham risk equations were used to estimate baseline cardiovascular event hazards that were subsequently multiplied by CKD-specific hazard ratios reflecting the increased risk of cardiovascular events independently associated with CKD stage [26, 33]. In one of these models [26], a relative risk reduction for myocardial infarction and stroke was applied to the baseline risk based on statin use. The relative risk reductions for CKD stage 3 ( $3 a$ and $3 b$ ) and stage 4 were obtained from a published meta-analysis [131] and the SHARP trial [41], respectively. The authors assumed that statins did not reduce cardiovascular risks for patients with CKD stage 5 . The second publication, describing an adapted version of the SHARP CKD-CVD policy model [33], included CVD as a separate sub-model, with health states including non-vascular death, vascular death, non-fatal major atherosclerotic event and non-fatal haemorrhagic stroke. Elbasha et al. [25] also used CKD stage-specific hazard rates for myocardial infarction and stroke, although they were applied to baseline probabilities reported in the underlying Framingham risk equation publications. Boersma et al. [23] used transition probabilities for cardiovascular event incidence that were estimated based on the PREVEND study [40]. In Howard et al. [36], patients had an annual probability of experiencing a fatal or non-fatal cardiovascular event based on clinical history (diabetes, hypertension, proteinuria) obtained from the general literature. Models of ESRD did not include CVD components.

In models focusing on patients with diabetic nephropathy, CVD was most commonly incorporated using risk equations or transition probabilities. Equations from the Framingham Heart Study [132, 133] or UKPDS [96, 134] were most commonly used to model cardiovascular events, including myocardial infarction and stroke. Transition probabilities were estimated based on data derived from various sources, including DCCT [125], MARVAL [135] and UKPDS [96, 134].

In addition to the models described, a further seven models of CVD were identified that included a CKD component [136-142]. An overview of the models is provided in Supplementary Tables 17-19 (Resource 7 of the ESM). In six of these models, CKD was incorporated through a single ESRD health state, and one model [141] included a sub-model 
for the progression of nephropathy. The main approach to modelling cardiovascular events by these models was to use transition probabilities. Probabilities were typically age and sex specific and derived from the Framingham risk equation or Framingham data/studies. Two models adjusted the risk of cardiovascular events for hypertension treatment but assumed such treatment had no comparable impact on the risk of CKD progression [138, 141].

\subsection{Time to and Time with ESRD}

Transition rates consistent with the core model structures presented in Figs. 2 and 3 were extracted from eligible studies and are summarised in Table 2. Overall, there was greater availability of transition rate data for model structures based on albuminuria than for CKD stage-based models defined by GFR, which would be expected given the greater number of diabetes models with CKD components identified. Significant variability was observed across several reported transition rates. Most notably, the annual transitions from CKD stage 3 to CKD stage 4 and the transitions from ESRD/ dialysis to death ranged from 0.008 to 0.405 and 0.008 to 0.626 , respectively. All extracted transition rates are summarised in Resource 8 in the ESM.

The mean rate of progression from CKD stage 4 to ESRD was greater than that from macroalbuminuria ( 0.067 vs. 0.043 , respectively); conversely, the mean rate of progression from CKD stage 4 to death was lower than that from macroalbuminuria ( 0.080 vs. 0.101 , respectively). As anticipated, across transition rates relating to model structures based on albuminuria, the rate of progression to death increased as disease severity increased, with a less clear pattern observed among transitions relating to a CKD stagebased model structure defined by GFR. Patients with ESRD/ dialysis were at the greatest risk of mortality (mean rate 0.177); following transplant, the rate of transition to death was comparable to that in earlier disease stages (mean rate 0.053).

In total, eight CKD models and 15 diabetes models with CKD components reported enough information to evaluate expected time to ESRD/death and/or time with ESRD. Significant parameter variability existed between models in both time to ESRD/death and time with ESRD, with only small improvements in consistency observed when considering just those models where starting health states were aligned (i.e. all patients initiated at CKD stage 1 or normoalbuminuria) (Fig. 4).

Such parameter variability was likely due to the heterogeneity in patient characteristics and disease severity across studies. The reported age of patients varied from 13 to 75 years, with most models evaluating a cohort aged between 30 and 50 years. The majority of CKD models initiated patients at CKD stage 1, with only two exceptions: Levy et al. [28] (CKD stage 3b/4) and Erickson et al. [26] (CKD stage 2). However, significant heterogeneity existed with respect to patients' disease background, the most notable of which were the models by Elbasha et al. [25] and Erickson et al. [26], which modelled cohorts with hepatitis $C$ and early autosomal dominant polycystic kidney disease, respectively. Among diabetes models with nephropathy, there was reasonable consistency with respect to starting health state, with only four of the 15 models not initiating patients in the state of normoalbuminuria. The most notable differences in disease background were the modelling of T1DM or T2DM, and within those settings, the choice of diabetes treatment.

Further, published models eligible for this analysis typically followed the core model structure identified earlier in this review, based on either CKD stage or albuminuria. Some variability existed with respect to explicitly incorporated health states outside of these core structures, where the number of health state transitions varied from two in the simplest model to 26 in the most complex. Such differences in health state definition likely influenced differences in progression estimates.

Mean time to ESRD/death was relatively consistent across CKD models and diabetes models with nephropathy. In CKD models, mean time to ESRD/death was 28.2 years when patients were initiated from the earliest CKD stage (21.6 years when initiating patients in the starting health state defined in the model publication) and 24.6 years in diabetes models with CKD components (24.0 years when initiating patients in the starting health state defined in the model publication). When evaluating time with ESRD, CKD models predicted extended ESRD survival over diabetes models with nephropathy (mean time with ESRD: 8.0 vs. 3.8 years).

\section{Discussion}

This review qualitatively describes how the progression of kidney disease was incorporated in economic models. Models focusing purely on CKD are typically based on (estimated or measured GFR) decline, with GFR progression predominantly based on a linear rate of decline, which could be adjusted for age, race, CKD stage, prevalence of proteinuria and clinical history (hypertension or diabetes). In comparison, diabetes models with nephropathy, where most of the economic models included nephropathy as a separate microvascular sub-model within the overall model, were most frequently based on the presence of albuminuria, where CKD progression was most commonly modelled with the use of transition probabilities calculated for progression between albuminuria health states. There were exceptions to these general rules, and the choice of modelling framework was likely driven by numerous factors, such as perspective, 
Table 2 Summary of annual transition rates reported in identified studies

\begin{tabular}{|c|c|c|c|c|c|c|}
\hline Transition & Mean & Median & Minimum & Maximum & $\begin{array}{l}\text { No. of } \\
\text { observations }\end{array}$ & References \\
\hline \multicolumn{7}{|l|}{ CKD transitions } \\
\hline CKD 1 to CKD 2 & 0.053 & 0.053 & 0.022 & 0.083 & 2 & {$[25,30]$} \\
\hline CKD 1 to death & 0.040 & 0.040 & 0.040 & 0.040 & 1 & {$[30]$} \\
\hline CKD 2 to CKD 3 & 0.071 & 0.054 & 0.002 & 0.175 & 4 & {$[25,27,30]$} \\
\hline CKD 2 to death & 0.027 & 0.027 & 0.003 & 0.051 & 2 & {$[27,30]$} \\
\hline CKD 3 to CKD 4 & 0.182 & 0.151 & 0.008 & 0.405 & 6 & {$[25,27,29,30]$} \\
\hline CKD 3 to death & 0.041 & 0.063 & 0.005 & 0.063 & 5 & {$[27,29,30]$} \\
\hline CKD 4 to CKD 5/ESRD & 0.067 & 0.059 & 0.010 & 0.148 & 12 & {$[25,27,29,30,53,54,58,61]$} \\
\hline CKD 4 to death & 0.080 & 0.062 & 0.008 & 0.177 & 10 & {$[27,29,30][58,61,66]$} \\
\hline \multicolumn{7}{|l|}{ Nephropathy-related transitions } \\
\hline No nephropathy to microalbuminuria & 0.028 & 0.027 & 0.003 & 0.060 & 24 & {$[23,74,79,88,92,98,103,111,117,141]$} \\
\hline No nephropathy to death & 0.026 & 0.006 & 0.000 & 0.194 & 16 & {$[23,74,79,80,88,92,113,117]$} \\
\hline Microalbuminuria to macroalbuminuria & 0.044 & 0.030 & 0.001 & 0.157 & 18 & {$[23,74,79,88,92,103,110,111,117]$} \\
\hline Microalbuminuria to death & 0.038 & 0.014 & 0.000 & 0.119 & 17 & {$[23,74,79,88,92,102,110,117]$} \\
\hline Macroalbuminuria to ESRD & 0.040 & 0.012 & 0.001 & 0.158 & 17 & {$[23,74,79,88,92,110,111,117]$} \\
\hline Macroalbuminuria to death & 0.101 & 0.086 & 0.007 & 0.289 & 15 & {$[23,74,79,88,92,110,117]$} \\
\hline \multicolumn{7}{|l|}{ ESRD transitions } \\
\hline ESRD/dialysis to transplant & 0.055 & 0.040 & 0.005 & 0.150 & 14 & {$[27,30,43,44,48,51,107,118,120]$} \\
\hline ESRD/dialysis to death & 0.177 & 0.146 & 0.008 & 0.626 & 56 & $\begin{array}{l}{[27,30,35,43-45,48,51,53,58,59,61,63} \\
\quad 66,74,79,88,92,94,98,107,110,111 \\
117,118,120,121,136,138,141]\end{array}$ \\
\hline Transplant to dialysis & 0.082 & 0.084 & 0.040 & 0.118 & 4 & {$[27,30,118]$} \\
\hline Transplant to death & 0.053 & 0.050 & 0.012 & 0.093 & 12 & {$[27,30,51,61,107,118,120]$} \\
\hline
\end{tabular}

Assumptions: Where relevant, transitions were matched to a baseline patient age of 50 years. Where ranges were reported, the midpoint of the range was extracted. Where male and female rates were reported, the average of the two was extracted. Duration of diabetes was assumed to be 10 years if not reported and required to calculate transition rates. All transitions are presented as annual probabilities. Depending on the model, ESRD includes non-dialysis ESRD and dialysis

$C K D$ chronic kidney disease, ESRD end-stage renal disease

Fig. 4 Predicted time to endstage renal disease (ESRD) or death and with ESRD. $C K D$ chronic kidney disease

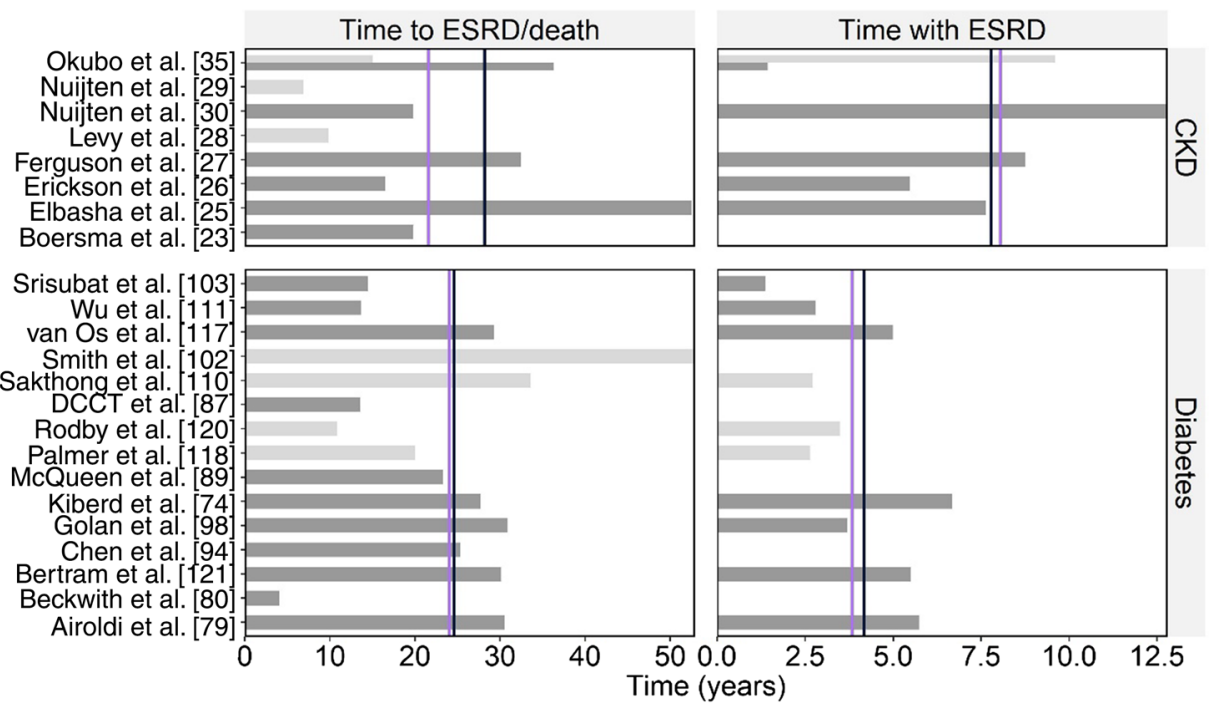

Mean time:

CKD stage $1 /$ Normoalbuminuria only

Starting health state:

All studies
CKD stage 1/Normoalbuminuria 
intervention, indication and underlying data sources used to inform disease progression, among other considerations.

The preference to model GFR over albuminuria (or vice versa) was not immediately clear and not typically justified in modelling publications; however, it may relate to the focus and structure of models in the two disease areas. For example, models focusing on CKD typically predicted health state occupancy in line with CKD stages in patients with established CKD, for which GFR provides an explicit delineator. In contrast, diabetes models with nephropathy typically aimed to predict the presence of ESRD based on a reported baseline prevalence of albuminuria, an established predictor of ESRD in patients with diabetes, often in patients with no history of CKD [143, 144]. However, while the choice of prognostic factor may be relatively straight forward within the context of specific modelling frameworks informed by the availability of data, the clinical rationale for any preference is much less well-defined [145]. Consensus does exist among the published literature with respect to the natural trajectory of albuminuria and GFR, with changes in albuminuria considered variable, with both negative and positive changes often observed in short timeframes, and changes in GFR usually considered to be progressive (i.e. monotonically decreasing). However, two studies predicted improvement in GFR and albuminuria health states [23, 27], in line with the literature suggesting that renal function can improve in a subset of patients [146-148]. Nevertheless, it has been suggested that the two variables be used in conjunction to predict the progression of CKD [145, 149].

There were some limitations to our review. First, only English articles were included in the search. However, this included the vast majority of the articles published, and research suggests that exclusion of non-English articles is unlikely to result in bias [150]. Second, only primary publications of models were included as they were deemed to contain the most relevant information regarding the model. Third, the review was limited to the information available in the study publication. Fourth, titles and abstracts of the identified citations were screened by a single reviewer. For uncertainties surrounding the relevance of a study, the corresponding full text was obtained, so a decision was made based on a thorough assessment of the study.

Across all models, a common author-identified limitation was the quality and quantity of the data sources that were used to inform the model, such as using data from clinical trials with a short duration, despite a longer follow-up study being more appropriate to model disease progression. For models of CKD without diabetes, limitations surrounded the modelling of CKD progression, including the lack of data for estimating the rate of decline in kidney function for people with neither diabetes nor hypertension [24], lack of stratification of CKD states according to albuminuria [25], and the assumption that GFR declines linearly [28]. In models of CKD with diabetes, a number of authors cited the use of risk equations derived from the Framingham Heart Study as a limitation to predicting cardiovascular events in those with CKD.

Interestingly, most models that captured the risk of CVD predicted an increase in cardiovascular risk as CKD progressed; conversely, only two models predicted an increased risk of CKD progression as a function of CVD incidence [23, 92]. However, it should be noted that the modelling of these components was often relatively simplistic and failed to provide clinical justification or rationale for the choice of modelled relationship. Across all models, cardiovascular-related risk was most frequently derived from the Framingham Heart Study, with estimates modified to reflect differences in specific geographical populations or patient characteristics. The Framingham risk equations are an extremely well-validated set of equations derived from a large cohort of patients (over multiple generations) followed-up over several decades and have helped identify major cardiovascular risk factors and their impact on the evolving risk of CVD [151-156]. However, the generalisability of the Framingham equations to a CKD population is uncertain, given the equations were derived using data from a population-based cohort with a low prevalence of CKD [157]. Various studies have demonstrated that patients with CKD have a higher prevalence of CVD than can reasonably be explained based on traditional cardiovascular risk factors, suggesting that CKD itself, or other unknown factors, are additionally impacting on patients' cardiovascular risk [3, 158, 159]. As a result, the Framingham risk equations demonstrate poor overall accuracy in predicting cardiovascular events in individuals with CKD, emphasising the need for the development of cardiovascular risk equations in patients with CKD specifically to support future cost-effectiveness studies [156].

Across models, CKD was generally described and modelled as a single disease, thus ignoring (perhaps relevant) differences in aetiologies. However, the interventions under evaluation were typically indicated across all CKD aetiologies and targeted to other diseases rather than to CKD itself, with decision makers most interested in an evaluation of cost effectiveness across the entire CKD population (cohort level as opposed to individual patient level). Further, while specific CKD aetiologies were not often defined or cited, modelled populations were usually defined based on an underlying clinical trial design, which typically limited patient heterogeneity through specific inclusion/exclusion criteria. Therefore, within this context, a simplification or generalisation of CKD aetiologies may be considered appropriate.

The clinical course of CKD progression (CKD stage 1-4) to ESRD is known to vary substantially across patients and may be influenced by various risk factors, including CKD aetiology, sex, presence of comorbidities (e.g. diabetes and 
CVD), anaemia, hypertension and proteinuria [160, 161]. Such heterogeneity in underlying disease poses challenges in the accurate modelling of CKD progression, and the models identified often did not consider these factors. Overall, the body of evidence on prognostic factors related to disease outcomes is relatively large in the field of diabetes, with a greater volume of prospective studies from which disease progression estimates can be derived or multivariable risk equations utilised. However, the tendency across all identified models was to capture input data at the mean rather than the observed patient-level variation, which often precluded the ability to capture true patient heterogeneity. More commonplace was the independent sampling of input data, through probabilistic sensitivity analysis to approximate heterogeneity, although this approach is associated with inherent limitations and does not actually capture how relevant differences in patients' clinical profiles affect costeffectiveness outcomes.

The time it takes a patient to reach ESRD/death, and the time for which a patient resides in the ESRD state, is of significance, particularly from the health economics perspective, since ESRD is associated with a significant resource burden and, consequently, may influence cost-effectiveness decisions. While there was general agreement amongst model type with respect to mean time to ESRD/death, mean time with ESRD was typically shorter among CKD models than among diabetes models with nephropathy. Such a difference may possibly be attributed to the excess morbidity and mortality associated with diabetes. In addition, a difference in the age distribution of patients may be a contributing factor. Overall, given the relatively large variations in time to and time with ESRD and their potential influence on health economics studies, we suggest that further research may be undertaken to fully quantify the differences across models' structures and better understand the heterogeneity between modelled populations and model designs, and the likely causes of such differences.

The heterogeneity with respect to both patient characteristics and disease outcome prediction, combined with a reasonable body of evidence, does seem to contribute to a wide use of microsimulation models (compared with Markov-based cohort models) in the field of diabetes. However, the rationale for model selection was often not cited in the various model publications identified in this review, and estimates of CKD progression in diabetes models with CKD components still rely heavily on a limited number of older datasets that may not fully capture contemporary patient care. Even though (at least) a similar level of patient and disease heterogeneity exists in the field of CKD, a Markov design was relatively common for simulating CKD progression. This may be driven by the limited data available to characterise the true variation in CKD progression patterns, although several risk equations are currently available [162].
It could be hypothesised that the economic modelling of CKD is less well-researched than that of diabetes because of the significant advancements in the treatment of diabetes over recent years and paralleled advancements in modelling methodologies. With the expected development of new CKD treatment options, we anticipate that our understanding of the most appropriate modelling methods for CKD will improve, with this review providing a basis from which further discussion may be considered.

\section{Conclusion}

Typically, GFR is used within CKD model frameworks and albuminuria in diabetes model frameworks. The prognostic ability of GFR to predict CKD progression is wellestablished in patients with CKD (both with and without diabetes). This, combined with the heterogenous nature of individual patients' GFR and albuminuria trajectories, provides rationale for a model structure designed around the prediction of individual patients' GFR trajectories. However, most models identified by this review were cohort based and/ or utilised input data at cohort mean levels, limiting the consideration of clinically relevant differences between patients' course of disease. Frameworks of future CKD models should be informed and justified based on clinical rationale and data availability to ensure validity of model results. In addition, further clinical and observational research is warranted to provide a better understanding of prognostic factors and data sources to improve economic modelling accuracy in CKD.

Author Contributions DS led the design and implementation of the research and the writing of the manuscript and undertook the systematic literature review. TW contributed to the design and implementation of the research, the analysis of the data and the writing of the manuscript. SR led and undertook the systematic literature review and contributed to the writing of the manuscript. PM provided senior review and technical expertise and contributed to the design of the research. HVH provided senior review, contributed to the design and implementation of the research and supported manuscript development. All named authors meet the International Committee of Medical Journal Editors (ICMJE) criteria for authorship for this manuscript, take responsibility for the integrity of the work as a whole, and have given final approval to the version to be published. As guarantor and corresponding author, DS takes full responsibility for the work as a whole and the decision to submit and publish the manuscript.

Data Availability All data generated or analysed during this study are included in this published article and the Supplementary Material.

\section{Compliance with Ethical Standards}

Funding Sponsorship for this work and article processing charges were funded by AstraZeneca.

Conflict of interest DS, TW, SR and PM are employees of Health Economics and Outcomes Research Ltd. and received funding from 
AstraZeneca to undertake the research outlined in this study. HVH is an employee of AstraZeneca.

Open Access This article is distributed under the terms of the Creative Commons Attribution-NonCommercial 4.0 International License (http://creativecommons.org/licenses/by-nc/4.0/), which permits any noncommercial use, distribution, and reproduction in any medium, provided you give appropriate credit to the original author(s) and the source, provide a link to the Creative Commons license, and indicate if changes were made.

\section{References}

1. Naghavi M, et al. Global, regional, and national age-sex specific mortality for 264 causes of death, 1980-2016: a systematic analysis for the Global Burden of Disease Study 2016. Lancet. 2017;390(10100):1151-210.

2. Ritz E, McClellan WM. Overview: increased cardiovascular risk in patients with minor renal dysfunction: an emerging issue with far-reaching consequences. J Am Soc Nephrol. 2004;15(3):513-6.

3. Go AS, et al. Chronic kidney disease and the risks of death, cardiovascular events, and hospitalization. N Engl J Med. 2004;351(13):1296-305.

4. Matsushita K, et al. Association of estimated glomerular filtration rate and albuminuria with all-cause and cardiovascular mortality in general population cohorts: a collaborative meta-analysis. Lancet. 2010;375(9731):2073-81.

5. Lawson CA, et al. Chronic kidney disease, worsening renal function and outcomes in a heart failure community setting: a UK national study. Int J Cardiol. 2018;267:120-7.

6. Liu M, et al. Cardiovascular disease and its relationship with chronic kidney disease. Eur Rev Med Pharmacol Sci. 2014;18(19):2918-26.

7. Mills KT, et al. A systematic analysis of worldwide populationbased data on the global burden of chronic kidney disease in 2010. Kidney Int. 2015;88(5):950-7.

8. Couser WG, et al. The contribution of chronic kidney disease to the global burden of major noncommunicable diseases. Kidney Int. 2011;80(12):1258-70.

9. Eggers PW. Has the incidence of end-stage renal disease in the USA and other countries stabilized? Curr Opin Nephrol Hypertens. 2011;20(3):241-5.

10. Rajan M, et al. Estimating utilities for chronic kidney disease, using SF-36 and SF-12-based measures: challenges in a population of veterans with diabetes. Qual Life Res. 2013;22(1):53-64.

11. Vanholder R, et al. Reducing the costs of chronic kidney disease while delivering quality health care: a call to action. Nat Rev Nephrol. 2017;13(7):393-409.

12. Braun L, et al. High burden and unmet patient needs in chronic kidney disease. Int J Nephrol Renovasc Dis. 2012;5:151-63.

13. Meguid El Nahas A, Bello AK. Chronic kidney disease: the global challenge. Lancet. 2005;365(9456):331-40.

14. James MT, Hemmelgarn BR, Tonelli M. Early recognition and prevention of chronic kidney disease. Lancet. 2010;375(9722):1296-309.

15. Piepoli MF, et al. 2016 European Guidelines on cardiovascular disease prevention in clinical practiceThe Sixth Joint Task Force of the European Society of Cardiology and Other Societies on Cardiovascular Disease Prevention in Clinical Practice (constituted by representatives of 10 societies and by invited experts)Developed with the special contribution of the European
Association for Cardiovascular Prevention \&amp; Rehabilitation (EA. Eur Heart J. 2016;37(29):2315-81.

16. World Health Organisation. Prevention of cardiovascular disease. 2007 22/03/2018]. http://www.who.int/cardiovascular_diseases/ guidelines/PocketGL.ENGLISH.AFR-D-E.rev1.pdf?ua=1.

17. Jones-Burton $\mathrm{C}$, et al. An in-depth review of the evidence linking dietary salt intake and progression of chronic kidney disease. Am J Nephrol. 2006;26(3):268-75.

18. Navaneethan SD, et al. Weight loss interventions in chronic kidney disease: a systematic review and meta-analysis. Clin J Am Soc Nephrol. 2009;4(10):1565-74.

19. Wakasugi M, et al. A combination of healthy lifestyle factors is associated with a decreased incidence of chronic kidney disease: a population-based cohort study. Hypertens Res. 2013;36(4):328-33.

20. Onuigbo MAC, Agbasi N. Chronic kidney disease prediction is an inexact science: the concept of "progressors" and "nonprogressors". World J Nephrol. 2014;3(3):31.

21. Moher D, et al. Preferred reporting items for systematic review and meta-analysis protocols (PRISMA-P) 2015 statement. Syst Rev. 2015;4:1.

22. Husereau, D., et al. Consolidated Health Economic Evaluation Reporting Standards (CHEERS) statement. 2013. 346: p. f1049.

23. Boersma $\mathrm{C}$, et al. Screen-and-treat strategies for albuminuria to prevent cardiovascular and renal disease: cost-effectiveness of nationwide and targeted interventions based on analysis of cohort data from the Netherlands. Clin Ther. 2010;32(6):1103-21.

24. Boulware LE, et al. Screening for proteinuria in US adults: a cost-effectiveness analysis. Jama. 2003;290(23):3101-14.

25. Elbasha E, et al. Cost-effectiveness of elbasvir/grazoprevir use in treatment-naive and treatment-experienced patients with hepatitis $\mathrm{C}$ virus genotype 1 infection and chronic kidney disease in the United States. J Viral Hepat. 2017;24(4):268-79.

26. Erickson KF, et al. Cost-effectiveness of statins for primary cardiovascular prevention in chronic kidney disease. J Am Coll Cardiol. 2013;61(12):1250-8.

27. Ferguson TW, et al. Screening for chronic kidney disease in Canadian indigenous peoples is cost-effective. Kidney Int. 2017;92(1):192-200.

28. Levy AR, et al. An epidemiologic model to project the impact of changes in glomerular filtration rate on quality of life and survival among persons with chronic kidney disease. Int J Nephrol Renovasc Dis. 2014;7:271-80.

29. Nuijten M, et al. Chronic kidney disease Markov model comparing paricalcitol to calcitriol for secondary hyperparathyroidism: a US perspective. Curr Med Res Opin. 2009;25(5):1221-34.

30. Nuijten M, et al. Cost effectiveness of paricalcitol versus a nonselective vitamin D receptor activator for secondary hyperparathyroidism in the UK: a chronic kidney disease markov model. Clin Drug Investig. 2010;30(8):545-57.

31. Orlando LA, et al. The chronic kidney disease model: a general purpose model of disease progression and treatment. BMC Med Inform Decis Mak. 2011;11(1):41.

32. Schlackow, I., et al., A policy model of cardiovascular disease in moderate-to-advanced chronic kidney disease. Heart. 2017: p. heartjnl-2016-310970.

33. Hoerger TJ, et al. A health policy model of CKD: 1. Model construction, assumptions, and validation of health consequences. Am J Kidney Dis. 2010;55(3):452-62.

34. Inker LA, et al. KDOQI US commentary on the 2012 KDIGO clinical practice guideline for the evaluation and management of CKD. Am J Kidney Dis. 2014;63(5):713-35.

35. Okubo R, et al. Cost-effectiveness of obstructive sleep apnea screening for patients with diabetes or chronic kidney disease. Sleep Breath. 2015;19(3):1081-92. 
36. Howard K, et al. Cost-effectiveness of screening and optimal management for diabetes, hypertension, and chronic kidney disease: a modeled analysis. Value Health. 2010;13(2):196-208.

37. Orlando LA, Owen WF, Matchar DB. Relationship between nephrologist care and progression of chronic kidney disease. NC Med J. 2007;68(1):9-16.

38. Levey AS, et al. A new equation to estimate glomerular filtration rate. Ann Intern Med. 2009;150(9):604-12.

39. Keith, D., et al. Mortality of chronic kidney disease (CKD) in a large HMO population. In Journal of the American Society of Nephrology. 2002. Lippincott Williams \& Wilkins 530 Walnut St, Philadelphia, PA 19106-3621 USA.

40. Hillege HL, et al. Microalbuminuria is common, also in a nondiabetic, nonhypertensive population, and an independent indicator of cardiovascular risk factors and cardiovascular morbidity. J Intern Med. 2001;249(6):519-26.

41. Baigent $\mathrm{C}$, et al. The effects of lowering LDL cholesterol with simvastatin plus ezetimibe in patients with chronic kidney disease (Study of Heart and Renal Protection): a randomised placebo-controlled trial. Lancet. 2011;377(9784):2181-92.

42. Beby AT, et al. Cost-effectiveness of high dose hemodialysis in comparison to conventional in-center hemodialysis in the Netherlands. Adv Ther. 2016;33(11):2032-48.

43. Gonzalez-Perez JG, et al. Hemodialysis for end-stage renal disease: a cost-effectiveness analysis of treatment options. Int $\mathbf{J}$ Technol Assess Health Care. 2005;21(1):32-9.

44. Howard $\mathrm{K}$, et al. The cost-effectiveness of increasing kidney transplantation and home-based dialysis. Nephrology (Carlton). 2009; 14(1):123-32.

45. Kirby L, Vale L. Dialysis for end-stage renal disease. Determining a cost-effective approach. Int J Technol Assess Health Care. 2001;17(2):181-9.

46. Lee CP, Chertow G, Zenios SA. A simulation model to estimate the cost and effectiveness of alternative dialysis initiation strategies. Med Decis Mak. 2006;26(2):535-49.

47. Liu FX, et al. High-dose hemodialysis versus conventional incenter hemodialysis: a cost-utility analysis from a UK payer perspective. Value Health. 2015;18(1):17-24.

48. Pike E, et al. More use of peritoneal dialysis gives significant savings: a systematic review and health economic decision model. J Clin Med Res. 2017;9(2):104-16.

49. Sennfalt K, Magnusson M, Carlsson P. Comparison of hemodialysis and peritoneal dialysis - a cost-utility analysis. Perit Dial Int. 2002;22(1):39-47.

50. Teerawattananon Y, Mugford M, Tangcharoensathien V. Economic evaluation of palliative management versus peritoneal dialysis and hemodialysis for end-stage renal disease: evidence for coverage decisions in Thailand. Value Health. 2007;10(1):61-72.

51. Yang F, Lau T, Luo N. Cost-effectiveness of haemodialysis and peritoneal dialysis for patients with end-stage renal disease in Singapore. Nephrology (Carlton). 2016;21(8):669-77.

52. Manns B, et al. Economic evaluation of sevelamer in patients with end-stage renal disease. Nephrol Dial Transplant. 2007;22(10):2867-78.

53. Adarkwah C, Gandjour A. Cost-effectiveness of angiotensin-converting enzyme inhibitors in nondiabetic advanced renal disease (Structured abstract). Expert Rev Pharmacoecon Outcomes Res. 2011;11(2):215-23.

54. Adarkwah CC, et al. To treat or not to treat? Cost-effectiveness of ace inhibitors in non-diabetic advanced renal disease-a Dutch perspective. Kidney Blood Press Res. 2013;37(2-3):168-80.

55. Clement FM, et al. An economic evaluation of erythropoiesis-stimulating agents in CKD. Am J Kidney Dis. 2010;56(6):1050-61.
56. Dany A, et al. Using repeated-prevalence data in multistate modeling of renal replacement therapy. J Appl Stat. 2015;42(5-6):1278-90.

57. de Wit GA, Ramsteijn PG, de Charro FT. Economic evaluation of end stage renal disease treatment. Health Policy. 1998;44(3):215-32.

58. Hiremath, S., G. Knoll, and M.C. Weinstein, Should the arteriovenous fistula be created before starting dialysis?: a decision analytic approach. PLoS One. 2011. 6(12).

59. Kiberd BA. A patient centered approach to the treatment of renal vascular disease to prevent end stage renal failure. Geriatr Nephrol Urol. 1997;7(2):61-6.

60. Levy AR, et al. Projecting long-term graft and patient survival after transplantation. Value Health. 2014;17(2):254-60.

61. Littlewood KJ, et al. Adjunctive treatment with moxonidine versus nitrendipine for hypertensive patients with advanced renal failure: a cost-effectiveness analysis. BMC Nephrol. 2007;8:9.

62. Naci H, et al. Historical clinical and economic consequences of anemia management in patients with end-stage renal disease on dialysis using erythropoietin stimulating agents versus routine blood transfusions: a retrospective cost-effectiveness analysis. J Med Econ. 2012;15(2):293-304.

63. Nuijten $\mathrm{M}$, et al. Health economic evaluation of paricalcitol((R)) versus cinacalcet + calcitriol (oral) in Italy. Clin Drug Investig. 2015;35(4):229-38 (corrected).

64. Nguyen HV, Bose S, Finkelstein E. Incremental cost-utility of sevelamer relative to calcium carbonate for treatment of hyperphosphatemia among pre-dialysis chronic kidney disease patients. BMC Nephrol. 2016;17(1):45.

65. Rosselli D, Rueda JD, Diaz CE. Cost-effectiveness of kidney transplantation compared with chronic dialysis in end-stage renal disease. Saudi J Kidney Dis Transpl. 2015;26(4):733-8.

66. Takahashi T, Reed SD, Schulman KA. Cost-effectiveness of the oral adsorbent AST-120 versus placebo for chronic kidney disease. Nephrology (Carlton). 2008;13(5):419-27.

67. Thaweethamcharoen $\mathrm{T}$, et al. Cost-utility analysis of erythropoietin for anemia treatment in thai end-stage renal disease patients with hemodialysis. Value in Health Reg Issues. 2014;3(1):44-9.

68. Thompson M, et al. Economic evaluation of sevelamer for the treatment of hyperphosphatemia in chronic kidney disease patients not on dialysis in the United Kingdom. J Med Econ. 2013;16(6):744-55.

69. Vegter S, et al. Cost-effectiveness of lanthanum carbonate in the treatment of hyperphosphatemia in chronic kidney disease before and during dialysis. Value Health. 2011;14(6):852-8.

70. Villa G, et al. Cost-effectiveness analysis of timely dialysis referral after renal transplant failure in Spain. BMC Health Serv Res. 2012;12:257.

71. de Vries EF, Rabelink TJ, van den Hout WB. Modelling the cost-effectiveness of delaying end-stage renal disease. Nephron. 2016;133(2):89-97.

72. Vegter $\mathrm{S}$, et al. The influence of future unrelated costs on costeffectiveness estimates: treatment of hyperphosphatemia with lanthanum carbonate in pre-dialysis patients with chronic kidney disease. Value Health. 2010;13(7):A478.

73. Shechter SM, et al. Cost-effectiveness analysis of vascular access referral policies in CKD. Am J Kidney Dis. 2017;70(3):368-76.

74. Kiberd BA, Jindal KK. Screening to prevent renal failure in insulin dependent diabetic patients: an economic evaluation. Bmj. 1995;311(7020):1595-9.

75. Renal Association. UK Renal Registry.

76. The Norwegian Renal Registry. Annual reports 2013. 2013.

77. de Charro FT, Ramsteyn PG. RENINE, a relational registry. Nephrol Dial Transplant. 1995;10(4):436-41.

78. U.S. Renal Data System, USRDS 2003 Annual Data Report: Atlas of End-Stage Renal Disease in the United States. 2003, 
National Institute of Health, National Institute of Diabetes and Digestive and Kidney Diseases: Bethesda, MD.

79. Airoldi M, et al. Requisite models for strategic commissioning: the example of type 1 diabetes. Health Care Manag Sci. 2008;11(2):89-110.

80. Beckwith J, et al. A health economic analysis of clinical islet transplantation. Clin Transplant. 2012;26(1):23-33.

81. Dong FB, et al. Cost effectiveness of ACE inhibitor treatment for patients with type 1 diabetes mellitus. Pharmacoeconomics. 2004;22(15):1015-27.

82. Garattini L, et al. Economic evaluation of ACE inhibitor treatment of nephropathy in patients with insulin-dependent diabetes mellitus in Italy. Pharmacoeconomics. 1997;12(1):67-75.

83. McEwan P, et al. The health economic value of changes in glycaemic control, weight and rates of hypoglycaemia in type 1 diabetes mellitus. PLoS One. 2016;11(9):e0162441.

84. McEwan P, et al. Evaluation of the cost-effectiveness of insulin glargine versus NPH insulin for the treatment of type 1 diabetes in the UK. Curr Med Res Opin. 2007;23(sup1):S7-19.

85. Palmer A, et al. The cost-effectiveness of different management strategies for type I diabetes: a Swiss perspective. Diabetologia. 2000;43(1):13-26.

86. Shearer A, et al. Cost-effectiveness of flexible intensive insulin management to enable dietary freedom in people with type 1 diabetes in the UK. Diabet Med. 2004;21(5):460-7.

87. The Diabetes Control and Complications Trial Research Group. Lifetime benefits and costs of intensive therapy as practiced in the Diabetes Control and Complications Trial. Jama. 1996;276:1409-15.

88. Thokala P, et al. Assessing the cost-effectiveness of type 1 diabetes interventions: the Sheffield Type 1 Diabetes Policy Model. Diabet Med. 2014;31(4):477-86.

89. McQueen RB, et al. Cost-effectiveness of continuous glucose monitoring and intensive insulin therapy for type 1 diabetes. Cost Effect Resour Allocat. 2011;9(1):13.

90. Bagust A, et al. An economic model of the long-term health care burden of type II diabetes. Diabetologia. 2001;44(12):2140-55.

91. Brown JB, et al. The global diabetes model: user friendly version 30. Diabetes Res Clin Pract. 2000;50:S15-46.

92. Campbell HM, et al. Pharmacoeconomic analysis of angiotensinconverting enzyme inhibitors in type 2 diabetes: a Markov model. Ann Pharmacother. 2007;41(7-8):1101-10.

93. Caro JJ, et al. Economic assessment of troglitazone as an adjunct to sulfonylurea therapy in the treatment of type 2 diabetes. Clin Ther. 2000;22(1):116-27.

94. Chen TH-H, Yen M-F, Tung T-H. A computer simulation model for cost-effectiveness analysis of mass screening for type 2 diabetes mellitus. Diabetes Res Clin Pract. 2001;54:37-42.

95. Chen J, et al. Development of a diabetes treatment simulation model: with application to assessing alternative treatment intensification strategies on survival and diabetes-related complications. Diabetes Obes Metab. 2008;10(s1):33-42.

96. Clarke P, et al. A model to estimate the lifetime health outcomes of patients with type 2 diabetes: the United Kingdom Prospective Diabetes Study (UKPDS) Outcomes Model (UKPDS no. 68). Diabetologia. 2004;47(10):1747-59.

97. Eastman RC, et al. Model of complications of NIDDM: I. Model construction and assumptions. Diabetes Care. 1997;20(5):725-34.

98. Golan L, Birkmeyer JD, Welch HG. The cost-effectiveness of treating all patients with type 2 diabetes with angiotensin-converting enzyme inhibitors. Ann Intern Med. 1999;131(9):660-7.

99. Hayes A, et al. UKPDS outcomes model 2: a new version of a model to simulate lifetime health outcomes of patients with type 2 diabetes mellitus using data from the 30 year United
Kingdom Prospective Diabetes Study: UKPDS 82. Diabetologia. 2013;56(9):1925-33.

100. Hoerger TJ, et al. Screening for type 2 diabetes mellitus: a costeffectiveness analysis. Ann Intern Med. 2004;140(9):689-99.

101. McEwan P, et al. Evaluation of the costs and outcomes from changes in risk factors in type 2 diabetes using the Cardiff stochastic simulation cost-utility model (DiabForecaster). Curr Med Res Opin. 2006;22(1):121-9.

102. Smith DG, et al. Markov modeling analysis of health and economic outcomes of therapy with valsartan versus amlodipine in patients with type 2 diabetes and microalbuminuria. J Manag Care Pharm. 2004;10(1):26-32.

103. Srisubat A, et al. Cost-effectiveness of annual microalbuminuria screening in Thai diabetics. Asian Biomed. 2014;8(3):371-9.

104. Steen Carlsson K, Persson U. Cost-effectiveness of add-on treatments to metformin in a Swedish setting: liraglutide vs sulphonylurea or sitagliptin. J Med Econ. 2014;17(9):658-69.

105. The CDC Diabetes Cost-effectiveness Group. Cost-effectiveness of intensive glycemic control, intensified hypertension control, and serum cholesterol level reduction for type 2 diabetes. Jama. 2002;287(19):2542-51.

106. Willis M, Asseburg C, He J. Validation of economic and health outcomes simulation model of type 2 diabetes mellitus (ECHOT2DM). J Med Econ. 2013;16(8):1007-21.

107. Zhou $\mathrm{H}$, et al. A computer simulation model of diabetes progression, quality of life, and cost. Diabetes Care. 2005;28(12):2856-63

108. Adarkwah CC, Gandjour A. Cost-effectiveness of angiotensinconverting enzyme inhibitors and angiotensin II receptor blockers in newly diagnosed type 2 diabetes in Germany. Int J Technol Assess Health Care. 2010;26(1):62-70.

109. National Institute for Health and Care Excellence. Technology appraisal guidance [TA336]: Empagliflozin in combination therapy for treating type 2 diabetes. 2015 [cited 03/04/2018 03/04/2018]. https://www.nice.org.uk/guidance/ta336.

110. Sakthong $\mathrm{P}$, et al. Cost-effectiveness of using angiotensin-converting enzyme inhibitors to slow nephropathy in normotensive patients with diabetes type II and microalbuminuria. Nephrology. 2001;6(2):71-7.

111. Wu B, et al. Prevention of renal failure in Chinese patients with newly diagnosed type 2 diabetes: a cost-effectiveness analysis. J Diabetes Investig. 2018;9(1):152-61.

112. Eddy DM, Schlessinger L. Validation of the Archimedes diabetes model. Diabetes Care. 2003;26(11):3102-10.

113. Grima DT, Thompson MF, Sauriol L. Modelling cost effectiveness of insulin glargine for the treatment of type 1 and 2 diabetes in Canada. Pharmacoeconomics. 2007;25(3):253-66.

114. Mueller E, et al. Development and validation of the Economic Assessment of Glycemic Control and Long-Term Effects of diabetes (EAGLE) model. Diabetes Technol Ther. 2006;8(2):219-36.

115. Palmer AJ, et al. The CORE Diabetes Model: projecting longterm clinical outcomes, costs and costeffectiveness of interventions in diabetes mellitus (types 1 and 2) to support clini$\mathrm{cal}$ and reimbursement decision-making. Curr Med Res Opin. 2004;20(sup1):S5-26.

116. Rodby RA, et al. An economic analysis of captopril in the treatment of diabetic nephropathy. Diabetes Care. 1996;19(10):1051-61.

117. van Os N, et al. Diabetes nephropathy in the Netherlands: a cost effectiveness analysis of national clinical guidelines. Health Policy. 2000;51(3):135-47.

118. Palmer AJ, et al. Cost-effectiveness of early irbesartan treatment versus control (standard antihypertensive medications excluding ACE inhibitors, other angiotensin-2 receptor antagonists, and dihydropyridine calcium channel blockers) or late irbesartan 
treatment in patients with type 2 diabetes, hypertension, and renal disease. Diabetes Care. 2004;27(8):1897-903.

119. Palmer A, et al. Cost-consequence analysis in a French setting of screening and optimal treatment of nephropathy in hypertensive patients with type 2 diabetes. Diabetes Metab. 2006;32(1):69-76.

120. Rodby RA, et al. The cost-effectiveness of irbesartan in the treatment of hypertensive patients with type 2 diabetic nephropathy. Clin Ther. 2003;25(7):2102-19.

121. Bertram M, et al. Assessing the cost-effectiveness of drug and lifestyle intervention following opportunistic screening for prediabetes in primary care. Diabetologia. 2010;53(5):875-81.

122. Dall TM, et al. Value of lifestyle intervention to prevent diabetes and sequelae. Am J Prev Med. 2015;48(3):271-80.

123. Clark WF, et al. To pay or not to pay? A decision and cost-utility analysis of angiotensin-converting-enzyme inhibitor therapy for diabetic nephropathy. Can Med Assoc J. 2000;162(2):195-8.

124. Hayashino $Y$, et al. Cost-effectiveness of administering oral adsorbent AST-120 to patients with diabetes and advancestage chronic kidney disease. Diabetes Res Clin Pract. 2010;90(2):154-9.

125. Keen H. The Diabetes Control and Complications Trial (DCCT). Health Trends. 1994;26(2):41-3.

126. Lewis EJ, et al. The effect of angiotensin-convertingenzyme inhibition on diabetic nephropathy. N Engl J Med. 1993;329(20):1456-62.

127. Moss SE, Klein R, Klein B. The 14-year incidence of lowerextremity amputations in a diabetic population. The Wisconsin Epidemiologic Study of Diabetic Retinopathy. Diabetes Care. 1999;22(6):951-9.

128. Klein R, et al. The 10-year incidence of renal insufficiency in people with type 1 diabetes. Diabetes Care. 1999;22(5):743-51.

129. Ravid M, et al. Long-term stabilizing effect of angiotensin-converting enzyme inhibition on plasma creatinine and on proteinuria in normotensive type II diabetic patients. Ann Intern Med. 1993;118(8):577-81.

130. Ravid M, et al. Main risk factors for nephropathy in type 2 diabetes mellitus are plasma cholesterol levels, mean blood pressure, and hyperglycemia. Arch Intern Med. 1998;158(9):998-1004.

131. Navaneethan SD, et al., HMG CoA reductase inhibitors (statins) for people with chronic kidney disease not requiring dialysis. Cochrane Database Syst Rev. 2009(2): p. Cd007784.

132. Anderson KM, et al. Cardiovascular disease risk profiles. Am Heart J. 1991;121(1 Pt 2):293-8.

133. D'Agostino RB, et al. General cardiovascular risk profile for use in primary care. Circulation. 2008;117(6):743-53.

134. King P, Peacock I, Donnelly R. The UK Prospective Diabetes Study (UKPDS): clinical and therapeutic implications for type 2 diabetes. Br J Clin Pharmacol. 1999;48(5):643-8.

135. Viberti G, Wheeldon NM. Microalbuminuria reduction with valsartan in patients with type 2 diabetes mellitus. Circulation. 2002;106(6):672-8.

136. Axelrod DA, et al. Percutaneous stenting of incidental unilateral renal artery stenosis: decision analysis of costs and benefits. J Endovasc Ther. 2003;10(3):546-56.

137. Dorenkamp M, et al. Potential lifetime cost-effectiveness of catheter-based renal sympathetic denervation in patients with resistant hypertension. Eur Heart J. 2012;34(6):451-61.

138. Gandjour A, Stock S. A national hypertension treatment program in Germany and its estimated impact on costs, life expectancy, and cost-effectiveness. Health Policy. 2007;83(2):257-67.

139. Geisler BP, et al. Cost-effectiveness and clinical effectiveness of catheter-based renal denervation for resistant hypertension. J Am Coll Cardiol. 2012;60(14):1271-7.

140. Marra C, et al. Cost-effectiveness of pharmacist care for managing hypertension in Canada. Can Pharm J/Revue des Pharmaciens du Canada. 2017;150(3):184-97.
141. Saito I, et al. Cost-utility analysis of antihypertensive combination therapy in Japan by a Monte Carlo simulation model. Hypertension Res. 2008;31(7):1373-83.

142. Tajeu GS, et al. Cost-effectiveness of antihypertensive medication: exploring race and sex differences using data from the reasons for geographic and racial differences in stroke study. Med Care. 2017;55(6):552-60.

143. Bakris GL. Microalbuminuria: prognostic implications. Curr Opin Nephrol Hypertens. 1996;5(3):219-23.

144. Basi S, et al. Microalbuminuria in type 2 diabetes and hypertension: a marker, treatment target, or innocent bystander? Diabetes Care. 2008;31(Suppl 2):S194-201.

145. Polkinghorne KR. Estimated glomerular filtration rate versus albuminuria in the assessment of kidney function: what's more important? The clinical biochemist. Reviews. 2014;35(2):67-73.

146. Weis $\mathrm{L}$, et al. Renal function can improve at any stage of chronic kidney disease. PLoS One. 2013;8(12):e81835.

147. $\mathrm{Hu} \mathrm{B}$, et al. Kidney function can improve in patients with hypertensive CKD. J Am Soc Nephrol JASN. 2012;23(4):706-13.

148. Smith $\mathrm{M}$, et al. Change in albuminuria and risk of renal and cardiovascular outcomes: natural variation should be taken into account. Kidney Int Rep. 2018;3(4):939-49.

149. Hallan SI, et al. Combining GFR and albuminuria to classify CKD improves prediction of ESRD. J Am Soc Nephrol. 2009;20(5):1069-77.

150. Morrison A, et al. The effect of English-language restriction on systematic review-based meta-analyses: a systematic review of empirical studies. Int J Technol Assess Health Care. 2012;28(2):138-44

151. Hermansson J, Kahan T. Systematic review of validity assessments of Framingham risk score results in health economic modelling of lipid-modifying therapies in Europe. PharmacoEconomics. 2018;36(2):205-13.

152. Hua $X$, et al. Validation and recalibration of the Framingham cardiovascular disease risk models in an Australian Indigenous cohort. Eur J Prev Cardiol. 2017;24(15):1660-9.

153. Majed B, et al. External validation of the 2008 Framingham cardiovascular risk equation for CHD and stroke events in a European population of middle-aged men. The PRIME study. Prev Med. 2013;57(1):49-54.

154. Zomer E, et al., Validation of two Framingham cardiovascular risk prediction algorithms in an Australian population: the 'old' versus the 'new' Framingham equation. 2011. 18(1):115-120.

155. Hemann BA, Bimson WF, Taylor AJ. The Framingham Risk Score: an appraisal of its benefits and limitations. Am Heart Hosp J. 2007;5(2):91-6.

156. Weiner DE, et al. The Framingham predictive instrument in chronic kidney disease. J Am Coll Cardiol. 2007;50(3):217-24.

157. Chang A, Kramer H. Should eGFR and albuminuria be added to the Framingham Risk Score chronic kidney disease and cardiovascular disease risk prediction. Nephron Clin Pract. 2011;119(2):c171-8.

158. Herzog CA, et al. Cardiovascular disease in chronic kidney disease A clinical update from kidney disease: Improving Global Outcomes (KDIGO). Kidney Int. 2011;80(6):572-86.

159. Sarnak MJ, et al. Kidney disease as a risk factor for development of cardiovascular disease: a statement from the American Heart Association Councils on Kidney in Cardiovascular Disease, High Blood Pressure Research, Clinical Cardiology, and Epidemiology and Prevention. Hypertension. 2003;42(5):1050-65.

160. Tsai, W.-C., et al., Risk factors for development and progression of chronic kidney disease: a systematic review and exploratory meta-analysis. Medicine. 2016. 95(11).

161. Staples A, Wong C. Risk factors for progression of chronic kidney disease. Curr Opin Pediatr. 2010;22(2):161. 
162. Tangri N, Ferguson T, Komenda P. Pro: risk scores for chronic kidney disease progression are robust, powerful and ready for implementation. Nephrol Dial Transplant. 2017;32(5):748-51.

163. Hoerger TJ, et al. Cost-effectiveness of screening for microalbuminuria among African Americans. J Am Soc Nephrol. 2012;23(12):2035-41.
164. Nagy B, et al. Cost-effectiveness of a risk-based secondary screening programme of type 2 diabetes. Diabetes/Metab Res Rev. 2016;32(7):710-29. 